\title{
A Construction of an Averaged Representation of Human Cortical Gyri Using Non-linear Principal Component Analysis
}

\author{
G. Lohmann ${ }^{1}$, D.Y. von Cramon $^{1}$, and A.C.F. Colchester ${ }^{2}$ \\ 1 Max-Planck-Institute for Human Cognitive and Brain Sciences, Leipzig, Germany \\ 2 University of Kent at Canterbury, UK
}

\begin{abstract}
Because of the complex shape of human cortical gyri and great variation between individuals, development of effective representation schemes which allow establishment of correspondence between individuals, extraction of average structure of a population, and coregistration has proved very difficult. We introduce an approach which extracts line representations of gyri at different depths from high resolution MRI, labels main gyri semi-automatically, and extracts a template from a population using non-linear principal component analysis. The method has been tested on data from 96 healthy human volunteers. The model captures the most salient shape features of all major cortical gyri, and can be used for inter-subject registration, for investigating regionalized inter-subject variability, and for inter-hemispheric comparisons.
\end{abstract}

\section{Introduction}

The idea of constructing a model brain is not new. Perhaps the most well-known example is the Talairach brain atlas. While this atlas constituted a tremendous step forward because for the first time a generally accepted coordinate system was introduced, it also soon became clear that a number of problems still remain to be solved. In particular, the Talairach atlas 1] is based on one single brain so that it does not account for inter-individual anatomical variations. For this reason, more representative brain templates have been introduced [2]. Probabilistic atlases detailing anatomical structures were targeted by the ICBM consortium [3]. These templates provide presence probability maps of different structures. Transfer of labels between atlas and new subject requires co-registration of a T1-weighted MRI scan, that is already linked to the atlas labels, to the same type of MRI obtained in the new subject. Voxel-based co-registration of highly variable structures such as cortical sulci and gyri is imperfect. Averaging co-registered scans tends to blur the boundaries even of major sulci and gyri and generally does not allow accurate transfer of labels from atlas to subject. For example, a sulcus label from the atlas will frequently overlie a gyrus and vice versa.

An alternative and promising approach is to extract gyri by data-driven segmentation followed by object-based matching between subjects. Surface-based 
methods analyse cortical curvature and use geodesic distance metrics to define boundaries [4. However, manual selection of neighbouring sulcus-pairs was required to initiate segmentation of a gyrus, and did not resolve the difficulties of substantial inter-subject variation whereby a significant number of the gyri in one individual seem to have no clear matching gyri in another. In preliminary experiments we found a voxel-based method for extracting gyral cores [5] to be more robust and we based our approach on this.

Our framework allows matching of the important gyri across subjects. In addition to supporting automated labelling, the gyral representation should allow inter-subject registration and may be used for quantitative studies of intersubject variability and inter-hemispheric differences.

\section{Data and Pre-processing}

Our input data consisted of 96 T1-weighted MRI brain data of healthy human volunteers acquired on a 3-Tesla magnetic resonance scanner (Bruker Medspec 300) using a MDEFT pulse sequence 6. The within-plane spatial resolution was set to $1 \times 1 \mathrm{~mm}$, the between plane resolution was approximately $1.5 \mathrm{~mm}$. All data sets were rotated and shifted into a standard stereotactic coordinate system with the origin residing halfway between $\mathrm{CA}$ and $\mathrm{CP}$ [1. At the same time, the data sets were resampled so that isotropic voxels of size $1 \times 1 \times 1 \mathrm{~mm}^{3}$ resulted. After resampling, each data set consisted of 160 image slices that contained $160 \times 200$ voxels covering the entire brain. We restricted our attention to the large primary gyri that can be identified in almost all healthy individuals on the lateral aspect of hemispheres. Secondary, and particularly tertiary, cortical folds have a high degree of inter-subject variability so that it is unrealistic to derive a valid model for them.

\section{Methods}

In our algorithm, cortical folds are represented as 3D polygonal lines so that folds extracted from a group of individuals form a cloud of such lines. The central idea of our approach is to subject such data clouds to non-linear principal component analysis so that a principal curve for each major gyrus results. A principal curve captures the most salient shape features of a data cloud. Our gyral model is a collection of such principal curves. In the following, we will present this sequence of processing steps in more detail.

\subsection{Representation of Gyri Using 3D Polygonal Lines}

The initial steps for automatically extracting the polygonal line representation of gyral cores are similar to those described in [5]. In the current work, we extend this approach to include extraction of the deep white matter surface, which smoothly connects the fundi of the major sulci, and we express the depth of gyri as a proportion of the distance between the outer (closed) and inner (opened) 
white matter surfaces. The rationale behind this approach is that various regions of the brain show marked differences in the depth of the cortical foldings. For example, pre-frontal sulci are generally more shallow than parietal sulci. A relative measure of depth as introduced here allows us to incorporate all major folds into a common framework so that regional differences in sulcal depth play a lesser role.

The algorithm consists of the following steps (fig. 1):

1. White matter segmentation.

2. Extraction of a superficial white matter surface (corresponding to the arachnoid surface overlying the superficial grey matter) by 3D morphological closing of the segmented white matter [7], using a spherical $12 \mathrm{~mm}$ radius structuring element.

3. Extraction of the deep white matter surface using a morphological opening with a $8 \mathrm{~mm}$ diameter spherical structuring element.

4. 3D distance transform to define the depth from the closed white matter surface 8 .

5. 3D distance transform to define the distance from the opened white matter surface.

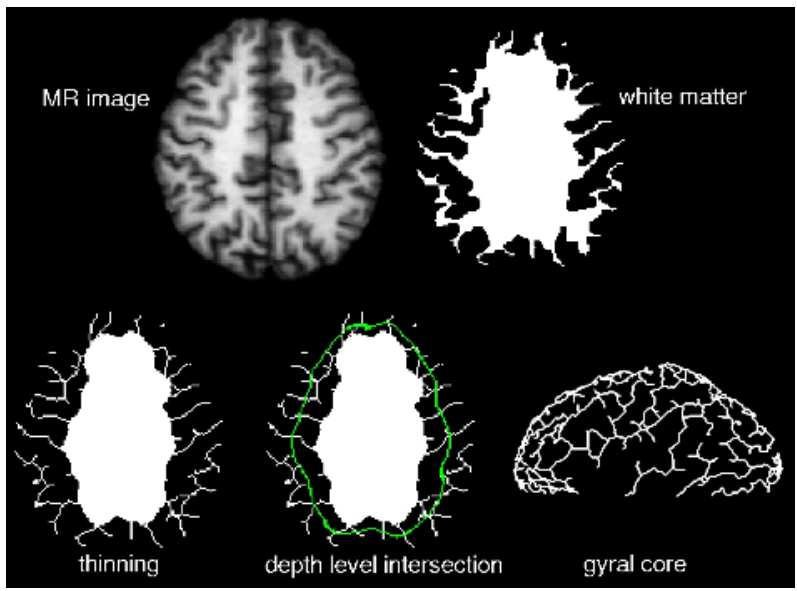

Fig. 1. The processing chain for obtaining the gyral core graph

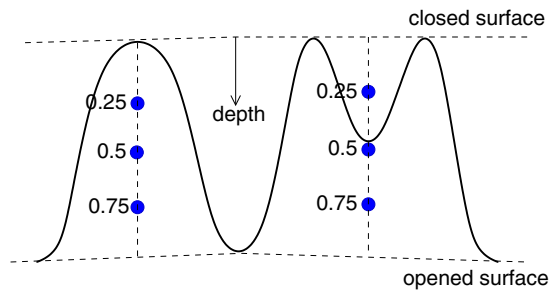

Fig. 2. Definition of relative depth 
6. Calculation of relative depth of any point as the ratio of the distance from the superficial surface to the sum of the two distances (to superficial and deep surfaces) (fig. 2).

7. Extraction of the gyri via a top-hat transformation (the white matter image minus the result of a $3 \mathrm{D}$ morphological opening).

8. 3D topological thinning of the gyri [9], 10].

9. Extraction of the intersections of the thinned gyri at a series of relative depths, to form "gyral cores".

10. Representation of the intersection as an undirected graph. The nodes in the graph correspond to voxels. Any two nodes are linked by an arc if their corresponding voxels are 26-connected.

We call our representation the "core graph". A path within the core graph is called a "gyral core". In the following, we will discuss some specific gyral cores that are of interest because of their interindividual consistency.

\subsection{Anatomical Labelling of Gyral Cores}

At the 0.95 depth level, gyri appear geometrically simple. They are smoother and less convoluted than at more shallow depth levels. This makes their identification much easier. Therefore, we initially attach anatomical labels only to gyral cores at the deepest depth level. These labels are then subsequently propagated upwards to all other levels of depth. We selected gyri which show high consistency across subjects to form the basis of the labelling scheme. These included the precentral, postcentral, middle frontal, parietal, inferior temporal, and superior temporal gyri (fig. 3). At the deepest relative depth levels, it was clear that the superior temporal gyral core was continuous with the opercular gyral bank

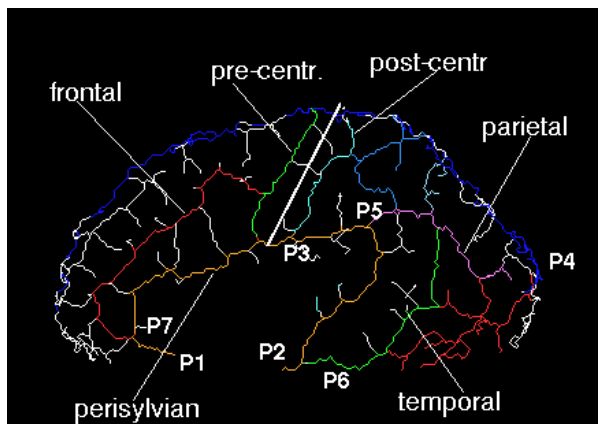

Fig. 3. Illustration of the gyral labelling procedure. Anchor points P1 and P2 mark the endpoints of the perisylvian core. The perisylvian core is defined as the shortest path in the core graph connecting P1 and P2. Other anchor points and the shortest paths between them are used for identifying other gyri. To identify the pre- and postcentral gyri, we additionally impose the constraint that the two gyri must be roughly parallel. 
above the circular sulcus of the insula, so we named this continuous gyral core perisylvian (fig. 3).

We devised a semi-automatic algorithm for labelling the above named gyral cores. For each of these six types of gyral cores we have devised a set of heuristic rules to be used in the identification process. Dijkstra's algorithm for finding shortest paths in graphs is one of the key elements of our method. For instance, to identify the perisylvian core, we first identify the two anchor points P1 and P2 that mark the anterior and posterior ends of this core. We then apply Dijkstra's algorithm for finding the shortest path in the core graph that connects P1 to P2. $\mathrm{P} 1$ is defined as the most ventral point whose $\mathrm{y}$-coordinate in the stereotactic Talairach system is less than -10. P2 is defined by a similar rationale.

Once the core graph of a data set is anatomically labelled at the deepest depth level, we can propagate its anatomical labels upwards to the next higher depth level, and so on, until the graph is labelled at all depths. This process is not very exact However, for the purpose of generating a generic model that is representative of 96 individuals, it is not essential to obtain a highly accurate labelling in each case.

\subsection{Principal Curves}

We have applied the anatomical labelling to all 96 data sets. In order to extract a generic gyral model from these data, we integrated the labelled gyral cores from all subjects into one data set using a common coordinate frame. We then extracted principal curves from this combined data set in order to reveal the generic structure of the data.

Principal curves are defined to be polygons which pass through the center of an n-dimensional data cloud. The data cloud is assumed to have an elongated shape so that a polygonal line can be considered to represent its most salient shape features. Its mathematical formulation is the following [11].

Let $X=\left\{x_{1}, \ldots, x_{n}\right\}, x_{i} \in R^{n}$ be a set of data points. A curve $f$ is called a principal curve of length $L$ for $X$ if $f$ minimizes

$$
\Delta(f)=E\left[\inf _{t}\|X-f(t)\|^{2}\right]
$$

the expected squared distance between $X$ and the curve, over all curves of length less than or equal to $L$. In [11], a suboptimal algorithm for learning principal curves from training data $X$ is described. The algorithm starts with a straight line segment that is iteratively refined as new vertices are added. With each addition of a new vertex, the resulting polygon achieves a more precise representation of the data. Figure 4 illustrates this process. For a more detailed description of the algorithm see [1].

This process was performed for each of the six anatomical labels and for each of the eight depth strata separately. More precisely, we extracted each of the six different type of anatomical labels from the combined data set in each depth level separately, and applied the principal curve extraction method described in [1]. 

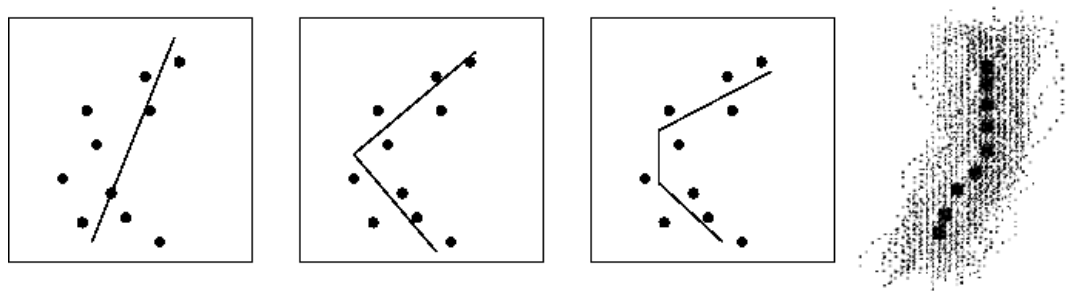

Fig. 4. Illustration of the principal curve algorithm [11. In the first iteration step, a straight line segment is placed such that it runs through the center of gravity of points projecting to it. In subsequent iterations, the shape representation is refined by adding more vertices. The rightmost image shows a principal curve running through the data cloud representing a post-central gyrus.

\section{Results}

We have applied the method for gyral core identification to all 96 data sets. In table 1, a list of the identification rates is given. Note that some types of gyral cores could be located in almost every data set while others were less robustly identified. The main reason for failure are topological interruptions of the gyral stalks because the algorithm relies on finding paths in the gyral graph. A disconnected gyral stalk produces a gap in the graph structure so that a 26connected path from one anchor point to the next does not exist. In cases where the automatic identification failed, it was supplied manually. In some regions especially in the anterior part of the middle frontal gyrus - the gyral core was only partially identifiable.

The resulting non-linear PCAs from eight depth levels were assembled into one output set to create the averaged representation of human cortical gyri (fig. 5). Note that the most shallow level of the representation is more convoluted than the deepest level (fig.5c,d). Furthermore, an inter-hemispheric difference emerges in the vicinity of the planum temporale. This region is connected to language processing, and it is well known that the left-hemispheric planum temporale is larger than the right [12].

Table 1. Identification accuracy of the gyral labelling. The numbers represent the absolute number of cases (out of a total of 96 data sets) in which the identification was successful. In some cases, gyral cores could be only partially identified (see text).

\begin{tabular}{|l|c|}
\hline Frontal, left & 94 \\
Frontal, right & 94 \\
Pre- and postcentral, left & 92 \\
Pre- and postcentral, right & 89 \\
\hline
\end{tabular}

\begin{tabular}{|l|l|}
\hline Perisylvian, left & 96 \\
Perisylvian, right & 96 \\
Temporal, left & 91 \\
Temporal, right & 95 \\
Parietal, left: & 95 \\
Parietal, right: & 95 \\
\hline
\end{tabular}




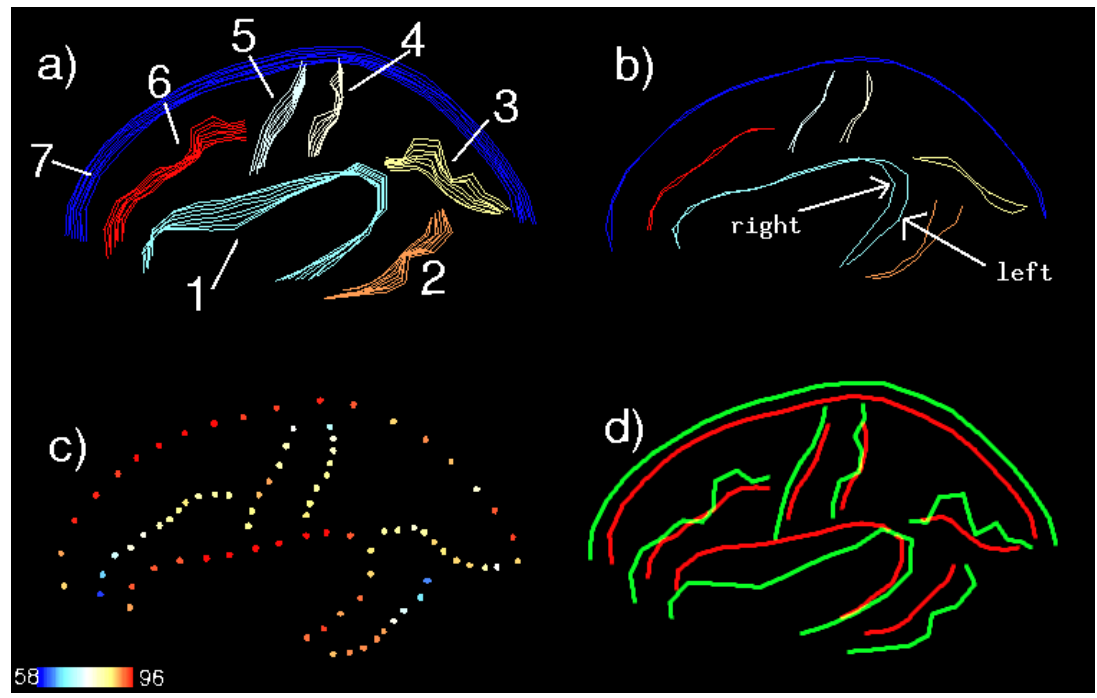

Fig. 5. The gyral model. 5a): the left hemisphere of the model in all its depth levels, 1:perisylvian, 2: inferior temporal, 3:parietal, 4:postcentral, 5:precentral, 6:frontal, 7:dorsal rim. 5b): an inter-hemispheric comparison where the right hemispheric model is flipped around the $\mathrm{x}$-axis and superimposed onto the left hemisphere. The arrows indicate a region of inter-hemispheric differences in the vicinity of the planum temporale. $5 \mathrm{c}$ ): the degree to which the model is representative of the population from which it was derived. Each node in the model graph has a label that indicates how many of the 96 inidividual labelled core graphs have a node of the same label within a $6 \mathrm{~mm}$ neighbourhood around the model node. Note that some segments of the model graph represent the data better than others. The anterior part of the middle frontal gyrus has low values indicating a high degree of inter-individual variability. Fig. 5d compares the deepest depth level of the model (red) with the most shallow level (green). Note that the gyri at the shallow level are more convoluted than at the deepest level.

\section{Discussion}

We have presented a method of constructing an averaged representation of human cortical gyri from a large population of individuals. We restrict the study to the gyri which are known from qualitative study of our data and from anatomical texts to be the most consistent. The method is based on measuring relative depth based on the deep white matter surface, on extraction of gyral cores which are 1-D structures in 3-D space at specific relative depths, and applying non-linear principal component analysis to the point sets to extract an average representation of the gyri. The model represents even quite subtle features of the cortical folding; for example, inter-hemispheric differences around the planum temporale are preserved. By comparing different strata of depth within the model, it is ev- 
ident that the cortical gyri generally become smoother and less convoluted with depth. However, this appears not to hold for the precentral gyrus. The model also highlights areas of high inter-individual variability such as the anterior part of the middle frontal gyrus.

In addition to allowing study of gyral variability across individuals and between hemispheres, the representation provides a framework which can be used for future work on non-rigid registration.

\section{References}

1. Talairach, P. Tournoux, J.: Co-planar Stereotaxic Atlas of the Human Brain: 3Dimensional Proportional System - an Approach to Cerebral Imaging. Thieme Medical Publishers, New York (1988)

2. Evans, D.L. Collins, S.R. Mills, E.D. Brown, R.L. Kelly, T.M. Peters, A.C.: 3d statistical neuroanatomical models from 305 mri volumes. In: Proc. IEEE-Nuclear Science Symposium and Medical Imaging Conference. (1993) 1813-1817

3. J.Mazziotta, A.Toga, A.Evans, M.Fox, J.Lancaster, K.Zilles, et al.: A probabilistic atlas and reference system for the human brain: International consortium for brain mapping (ICBM). Philosophical Trans. of the Royal Society: Biological Sciences 356 (2001) 1293-1322

4. Cachia, J.F. Mangin, D. Riviere, F. Kherif, N. Boddaert, A.Andrade, D. Papadopoulos-Orfanos, J.B. Poline, I. Bloch, M. Zilbovicius, P. Sonigo, F. Brunelle, J. Regis, A.: A primal sketch of the cortex mean curvature: A morphogenesis based approach to study the variability of the folding patterns. IEEE Transactions on Medical Imaging 22 (2003) 754-765

5. G. Lohmann: Extracting line representations of sulcal and gyral patterns in MR images of the human brain. IEEE Transactions on Medical Imaging 17 (1998) 1040-1048

6. Norris, D.: Reduced power multi-slice MDEFT imaging. J. Magn. Reson. Imaging 11 (2000) 445-451

7. Maragos, R.W. Schafer, P.: Morphological systems for multidimensional signal processing. Proc. of the IEEE 78 (1990) 690-709

8. Borgefors, G.: On digital distance transformations in three dimensions. Computer Vision, Graphics, and Image Processing: Image Inderstanding 64 (1996) 368-376

9. Tsao, K.S. Fu, Y.: A parallel thinning algorithm for 3D pictures. Computer Graphics Image Proc. 17 (1981) 315-331

10. A.Manzanera,T.Bernard,F.Preteux,B.Longuet: nD skeletonization: a unified mathematical framework. Journal of Electronic Engineering 11 (2002) 25-37

11. Kégl, B.: Learning and design of principal curves. IEEE Transactions on Pattern Analysis and Machine Intelligence 22 (2000) 281-297

12. Jancke, H. Steinmetz, L.: Auditory lateralization and planum temporale asymmetry. Neuroreport 5 (1993) 169-172 